\title{
THREE WEIL REPRESENTATIONS ASSOCIATE TO FINITE FIELDS
}

\author{
BY PAUL GÉRARDIN ${ }^{1}$ \\ Communicated by Robert M. Fossum, November 3, 1975
}

\begin{abstract}
Following the method of A. Weil [4], we define the Weil representation of general linear groups in 1 , of symplectic groups (odd characteristic) in 2 , of unitary groups in 3 , over finite fields. We give its character and decomposition and some functorial properties. The symplectic case was also studied by R. Howe [1] and M. Saito [3], the unitary case by R. I. Lehrer [2].
\end{abstract}

1. The Weil representations of symplectic groups (odd characteristic).

1.1. Let $(E, j)$ be a symplectic vector space over the field $k$ with $q$ elements. Let $H(E, j)$ be the group $E \times k$ with the law

$$
(w, z)\left(w^{\prime}, z^{\prime}\right)=\left(w+w^{\prime}, z+z^{\prime}+i\left(w, w^{\prime}\right)\right),
$$

where $i=j / 2$. It is a two-step nilpotent group with center $Z$ isomorphic to $k$ by $z \mapsto(0, z)$. The group $\operatorname{Sp}(E, j)$ of $j$ acts on $H(E, j)$ by $s:(w, z) \mapsto(s w, z)$.

1.2. For each nontrivial character $\zeta$ of $Z$, there is a unique class $\eta_{\xi}^{(E, j)}$ of irreducible representations of $H(E, j)$ given by $\zeta$ on $Z$.

THEOREM 1. There is a unique extension $W_{\xi}^{(E, j)}$ of $\eta_{\xi}^{(E, j)}$ to $\operatorname{Sp}(E, j)$, except for $q=3, \operatorname{dim} E=2$, where there is a unique extension $W_{\xi}^{(E, j)}$ disjoint from its conjugate.

The representation $W_{\xi}^{(E, j)}$ is called the Weil representation of $\operatorname{Sp}(E, j)$ associated to the character $\zeta$.

1.3. The Weil representations $W_{\xi}^{(E, j)}$ have the following properties:

(1) $W_{\zeta}^{(E, j)}=W_{\xi^{\prime}}^{(E, j)}$ iff $\zeta^{\prime}((0, z))=\zeta\left(\left(0, z t^{2}\right)\right)$ for a $t \in k^{*}$ and all $z \in k$.

(2) $W_{\xi}^{(E, j)}$ splits in two simple components of degree $\left(q^{n}+1\right) / 2$ and $\left(q^{n}-1\right) / 2$, where $n=\operatorname{dim} E$, given on the center of $\operatorname{Sp}(E, j)$ respectively by $(1 / q)^{n}$ and $-(1 / q)^{n}$.

(3) The complex conjugate of $W_{\zeta}^{(E, j)}$ is $W_{\zeta-1}^{(E, j)}$.

(4) The support of the character of $W_{\xi}^{(E, j)} \otimes \eta_{\xi}^{(E, j)}$ is the set of conjugates of $\operatorname{Sp}(E, j) Z$. $\mathbf{C}[E]$.

(5) The class $W_{\xi}^{(E, j)} \otimes W_{\zeta^{-1}}^{(E, j)}$ is the natural representation of $\operatorname{Sp}(E, j)$ in

AMS (MOS) subject classifications (1970). Primary 20C15, 20G40; Secondary $10 \mathrm{C} 30$.

${ }^{1}$ Supported in part by NSF Grant MPS72-05055A02. 
(6) For $s \in \operatorname{Sp}(E, j)$, $\operatorname{Tr} W_{\xi}^{(E, j)}(s)$ has $q^{N(s)}$ for absolute value, $2 N(s)=$ $\operatorname{dim} \operatorname{Ker}(s-1)$.

(7) Let $E_{1}$ be an isotropic subspace of $E$ and $E_{0}=E_{1}^{1} / E_{1}$. There is a natural surjective map from the stabilizer $L$ of $E_{1}$ in $\operatorname{Sp}(E, j) H(E, j)$ onto $\operatorname{Sp}\left(E_{0}, j_{0}\right) H\left(E_{0}, j_{0}\right)$, where $j_{0}$ is the symplectic form induced by $j$ on $E_{0}$. Let $P$ be the stabilizer of $E_{1}$ in $\operatorname{Sp}(E, j)$. The representation $\pi$ of $P H(E, j)$ induced by the representation $W_{\xi}^{\left(E_{0}, j_{0}\right)} \otimes \eta_{\xi}^{\left(E_{0}, j_{0}\right)}$ of $L$ is the product of the restriction

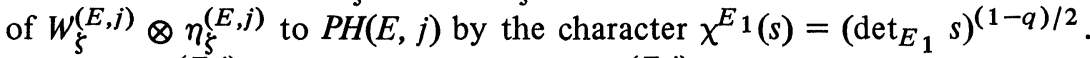
Moreover $W_{\xi}^{(E, j)}$ is the only extension of $\eta_{\xi}^{(E, j)}$ to $\operatorname{Sp}(E, j)$ which is given by $\chi^{E_{1}} \otimes \pi$ on $P$.

(8) If $E=\Sigma E_{r}$ (orthogonal sum), and $j_{r}$ is the restriction of $j$ to $E_{r}$, the restriction of $W_{\xi}^{(E, j)}$ to the product $\Pi \operatorname{Sp}\left(E_{r}, j_{r}\right)$ is $\bigotimes w_{\xi}^{\left(E_{r}, j_{r}\right)}$.

(9) If $E=\operatorname{Res}_{k^{\prime} / k} E^{\prime}$ and $j^{\prime}$ is a symplectic form on $E^{\prime}$ such that $j=$ $\operatorname{Tr}_{k^{\prime} / k^{j^{\prime}}}$, the restriction of $W_{\xi}^{(E, j)}$ to $\operatorname{Sp}\left(E^{\prime}, j^{\prime}\right)$ is $W_{\xi^{\prime}}^{\left(E^{\prime}, j^{\prime}\right)}$ where $\zeta^{\prime}\left(\left(0, z^{\prime}\right)\right)=$ $\zeta\left(\left(0, \operatorname{Tr}_{k^{\prime} / k} z^{\prime}\right)\right)$.

(10) If $t \in \operatorname{Sp}(E, j)$ is semisimple, $t$ belongs to a subgroup isomorphic to a product of $k_{r}( \pm)$, where $k_{r}(-)$ is the multiplicative group of the extension of degree $r$ of $k$, and $k_{r}(+)$ is the kernel of the norm from $k_{2 r}^{*}$ to $k_{r}^{*}$. The trace of $W_{\xi}^{(E, j)}$ on $t$ is $\chi(t)(-1)^{a(t)} q^{N(t)}$, with $\chi(t)=\Pi t_{r}^{\left(1 \pm q^{r}\right) / 2}$ where $t_{r}$ is the component of $t$ in $k_{r}( \pm), a(t)$ is the number of $r$ such that $t_{r} \neq 1$. The other elements of $\operatorname{Sp}(E, j)$ are in proper subgroups of type $P$ as in (7), and the character of $W^{(E, j)}$ on them is obtained from the formula of induced characters.

\section{The Weil representations of unitary groups.}

2.1. Let $K / k$ be a quadratic extension of the field $k$ with $q$ elements, $F$ a vector space over $K$ and $i$ a nondegenerate skew-hermitian form on $F$. The set of all couples $(w, z), w \in F, z \in K$ with $z-\bar{z}=i(w, w)$, is a group by the law (1); it is a two-step nilpotent group $H(F, i)$ with center $Z$ isomorphic to $k$ by $z$ $\mapsto(0, z)$. The group $U(F, i)$ of the form $i$ acts on $H(F, i)$ by $u$ : $(w, z) \mapsto$ $(u w, z)$.

2.2. For each nontrivial character $\zeta$ of the center $Z$ of $H(F, i)$ there is a unique class $\eta_{\xi}^{(F, i)}$ of irreducible representations of $H(F, i)$ given by $\zeta$ on $Z$.

THEOREM 2. There is a unique extension $W^{(F, i)}$ of $\eta_{\xi}^{(F, i)}$ to $U(F, i)$ such that:

(a) for $q$ odd, $W^{(F, i)}=\chi^{(F, i)} \otimes W_{\xi}^{(E, j)}$ on $U(F, i)$, where $\chi^{(F, i)}(u)=$ $(\operatorname{det} u)^{(1+q) / 2}, E$ is the underlying $k$-vector space of $F, j=\operatorname{Tr}_{K / k} i$, and $W_{\xi}^{(E, j)}$ is the Weil representation of $\operatorname{Sp}(E, j)$ associated to $\zeta$;

(b) for $q$ even, $W^{(F, i)}$ is real, and moreover for $q=2, \operatorname{dim} F=2, W^{(F, i)}$ contains no one dimensional representation of $U^{(F, i)}$ which factors through the determinant. The representation $W^{(F, i)}$ is called the Weil representation of $U(F, i)$.

2.3. The Weil representation $W^{(F, i)}$ has the following properties: 
(1) $W^{(F, i)}$ does not depend on $\zeta$.

(2) Let $n=\operatorname{dim}_{K} F ; W^{(F, i)}$ splits in $q$ simple classes of degree $\left[q^{n}-(-1)^{n}\right] /(q+1)$ corresponding to the nontrivial characters of the center of $U^{(F, i)}$ and, for $n>1$, a simple class of degree $q\left[q^{n-1}-(-1)^{n-1}\right] /(q+1)$.

(3) For $u \in U^{(F, i)}, \operatorname{Tr} W^{(F, i)}(u)=(-1)^{n}(-q)^{N(u)}$, where $N(u)=$ $\operatorname{dim}_{K} \operatorname{Ker}(u-1)$.

(4)-(10) As in part 1, (4)-(10) with the obvious modifications and all the characters $\chi$ are now trivial; in (9) $F^{\prime}=\operatorname{Res}_{K^{\prime} / K} F$ and $\left[K^{\prime}: K\right]$ is odd, $i=$ $\operatorname{Tr}_{K}^{\prime} / K i^{\prime}$.

3. The Weil representations of general linear groups. If the class $W^{V}$ of the natural representation of $\mathrm{GL}(V)$, for a finite dimensional vector space over the field $k$ of order $q$, in the space of complex functions on $V$ is called the Weil representation of GL(V), its properties are similar to those of the Weil representation of the unitary group of same rank over $k$ (for example, (2) and (4) up to the sign $\left.(-1)^{n}\right)$.

\section{BIBLIOGRAPHY}

1. R. Howe, On the character of Weil's representation, Trans. Amer. Math. Soc. 177 (1973), 287-298. MR 47 \#5180.

2. G. I. Lehrer, Weil representations and cusp-forms on unitary groups, Bull. Amer. Math. Soc. 80 (1974), 1137-1141.

3. M. Saito, Représentations unitaires des groupes symplectiques, J. Math. Soc. Japan 24 (1972), 232-251. MR 45 \#8776.

4. A. Weil, Sur certains groupes d'opérateurs unitaires, Acta Math. 111 (1964), 143211. MR 29 \#2324.

SCHOOL OF MATHEMATICS, INSTITUTE FOR ADVANCED STUDY, PRINCETON, NEW JERSEY 08540

UER DE MATHÉMATIQUES, UNIVERSITÉ PARIS VII, 2 PL. JUSSIEU, 75005 PARIS, FRANCE 